\title{
Tuberculosis of the head and neck - epidemiological and clinical presentation
}

\author{
Antoni Bruzgielewicz, Anna Rzepakowska, Ewa Osuch-Wójcikewicz, Kazimierz Niemczyk, \\ Rafał Chmielewski
}

Otolaryngology Department, Warsaw Medical University, Warsaw, Poland

Submitted: 22 December 2011

Accepted: 3 September 2012

Arch Med Sci 2014; 10, 6: 1160-1166

DOI: 10.5114/aoms.2013.34637

Copyright $\odot 2014$ Termedia \& Banach

\author{
Corresponding author: \\ Anna Rzepakowska MD, PhD \\ Otolaryngology Department \\ Warsaw Medical University \\ 1 a Banacha St \\ 02-097 Warsaw, Poland \\ Phone: +48606 831044 \\ E-mail: anula g@tlen.pl
}

\section{Abstract}

Introduction: The aim of our retrospective study was to review the clinical and epidemiological presentation of head and neck tuberculosis.

Material and methods: We analyzed the history of 73 patients with head and neck tuberculosis hospitalized in the Department of Otolaryngology, Medical University of Warsaw, between 1983 and 2009.

Results: We found that 26 (35.6\%) patients presented with lymph node tuberculosis, $20(27.4 \%)$ with laryngeal tuberculosis, $10(13.7 \%)$ with oropharyngeal tuberculosis, 9 (12.3\%) with salivary gland tuberculosis, 3 (4.1\%) with tuberculosis of paranasal sinuses, $3(4.1 \%)$ with aural tuberculosis, and $2(2.7 \%)$ with skin tuberculosis in the head and neck region. Within the group of patients with lymph node tuberculosis in 15 cases there were infected lymph nodes of the $2^{\text {nd }}$ and $3^{\text {rd }}$ cervical region and in 11 infected lymph nodes of the $1^{\text {st }}$ cervical region. In 5 cases of laryngeal tuberculosis there was detected coexistence of cancer. Oropharyngeal tuberculosis in 7 cases was localized in tonsils, where in 1 case coexisting cancer was diagnosed. Chest X-ray was performed in all cases and pulmonary tuberculosis was identified in 26 (35.6\%) cases.

Conclusions: We conclude that tuberculosis still remains a problem and must be taken into consideration in the diagnostic process. The coincidence of tuberculosis and cancer is remarkable in the head and neck region.

Key words: head and neck tuberculosis, lymph node, laryngeal, oropharyngeal tuberculosis.

\section{Introduction}

Tuberculosis is still one of the major causes of illness and death worldwide. According to the latest WHO report in 2006 there were 9.2 million new cases and 1.7 million deaths from tuberculosis, of which 0.7 million cases and 0.2 million deaths were in HIV-positive people [1]. Asia (SouthEast Asia and Western Pacific regions) and Africa account for more than $85 \%$ of global cases [1].

In the European Union countries the epidemiological situation of tuberculosis is satisfactory. In most of them the incidence of tuberculosis is less than 15 cases per 100000 people. But in Poland, Hungary, Portugal, Latvia, Estonia and Lithuania the incidence still exceeds the rate of 20 cases per 100000 people [2]. There were reported 22.5 new cases of tuberculosis per 100000 people in our country in 2006 [2]. 
Commonly known groups of high risk of tuberculosis are: people with HIV infection, people who had contact with a sputum positive patient with tuberculosis, people who abuse alcohol, drug addicts, homeless, immigrants from countries of high incidence of tuberculosis, patients with chronic diseases, for example diabetes, malignant neoplasm, heart insufficiency, renal insufficiency, different immunosuppressive diseases and treatments.

In $90 \%$ tuberculosis is located in the lungs. Extrapulmonary cases account for only $10 \%$. Head and neck region is not a common localization of tuberculosis; however, due to the great number of lymph nodes it is not casuistic and should be taken into consideration during the diagnostic process.

The aim of our study was to review the clinical and epidemiological presentation of tuberculosis in otolaryngology practice.

\section{Material and methods}

We analyzed retrospectively a group of 73 patients with tuberculosis of the head and neck region who presented at the Department of Otolaryngology, Medical University of Warsaw, between 1983 and 2009. Age and sex data, localization of the disease, status of contacts with active tuberculosis cases, and results of tuberculin skin tests were obtained and reviewed. Moreover radiological and histopathological findings were investigated. In all cases diagnosis was estimated by histopathological examination of infected tissue. For comprehensive diagnosis and treatment all patients were referred to one of the two specialist centers of tuberculosis in Warsaw.

The Bioethics Committee at the Medical University of Warsaw was informed about performed retrospective study and voiced no reservations.

In the study there were used basic statistical methods.

\section{Results}

In the group of 73 patients with head and neck tuberculosis there were 43 men and 30 women aged from 21 to 78 years (average 60.6 years). Age of men ranged from 36 to 75 years (average 62.5 years) and of women from 21 to 78 years (average 59.0 years). The male to female ratio was 1.43 .

The most common localization of tuberculosis in the head and neck region was observed to be cervical lymph nodes - 26 (35.6\%) cases. The second most frequent localization - 20 (27.4\%) cases - was laryngeal tuberculosis. In 10 (13.7\%) cases oropharyngeal tuberculosis was diagnosed. There were 9 cases of tuberculosis of salivary glands: $6(8.2 \%)$ cases of parotid gland tuberculosis and 3 (4.1\%) of submandibular gland tuberculosis.

Remaining cases comprised: tuberculosis of the paranasal sinuses - 3 (4.1\%) cases, aural tuberculosis - $3(4.1 \%)$ cases, skin tuberculosis in the head and neck region - 2 (2.7\%) cases. Detailed data of all patients are presented in Table I.

In the following paragraphs we present and discuss the patients according to the region of the head and neck affected.

\section{Cervical lymph node tuberculosis - 26 (35.6\%) cases}

The age range in this group was from 21 to 72 years. Mean age 56.7 years. There were 15 men (mean age: 59.7 years) and 11 women (mean age: 52.5 years). The mean time from the onset of symptoms to hospitalization was 17 weeks (range: 7-58 weeks). In 20 (76.9\%) cases the only complaint was the tumor on the neck. In 6 (23\%) cases patients additionally complained of systemic symptoms: malaise, fever and weight loss.

In 16 (61.5\%) cases there was lymphadenopathy on the left side, in 8 (30.8\%) cases on the right side and in $2(7.7 \%)$ cases bilaterally. Fifteen patients had enlarged lymph nodes of cervical regions II and III and 11 patients had enlarged lymph nodes of cervical region I.

The majority of patients presented with small to moderate size of lymphadenopathy (less than $3 \mathrm{~cm}$

Table I. Selected data of patients with head and neck tuberculosis

\begin{tabular}{|lcccc|}
\hline Localization of tuberculosis & Number (\%) & $\begin{array}{c}\text { Age range [years] } \\
\text { (mean age/SD) }\end{array}$ & $\begin{array}{c}\text { Sex: } \\
\text { male/female }\end{array}$ & $\begin{array}{c}\text { Period from onset } \\
\text { of symptoms } \\
\text { to hospitalization/ } \\
\text { mean [weeks] }\end{array}$ \\
\hline Cervical lymph nodes & $26(35.6)$ & $21-72(56.7 / 12.8)$ & $15 / 11$ & $7-58 / 17$ \\
\hline Larynx & $20(27.4)$ & $42-75(56.5 / 8.5)$ & $14 / 6$ & $10-63 / 22$ \\
\hline Oropharynx & $10(13.7)$ & $49-71(58.2 / 6.7)$ & $7 / 3$ & $6-25 / 14$ \\
\hline Salivary glands & $9(12.3)$ & $52-72(64.3 / 7.4)$ & $3 / 6$ & $14-36 / 20$ \\
\hline Nose and paranasal sinuses & $3(4.1)$ & $58-68(61.7 / 5.5)$ & $2 / 1$ & $8-18 / 14$ \\
\hline Ear & $3(4.1)$ & $52-71(61.3 / 9.5)$ & $2 / 1$ & $12-19 / 16$ \\
\hline Skin & $2(2.7)$ & $56-78(67 / 15.6)$ & $0 / 2$ & $0.5-8 / 4.25$ \\
\hline
\end{tabular}




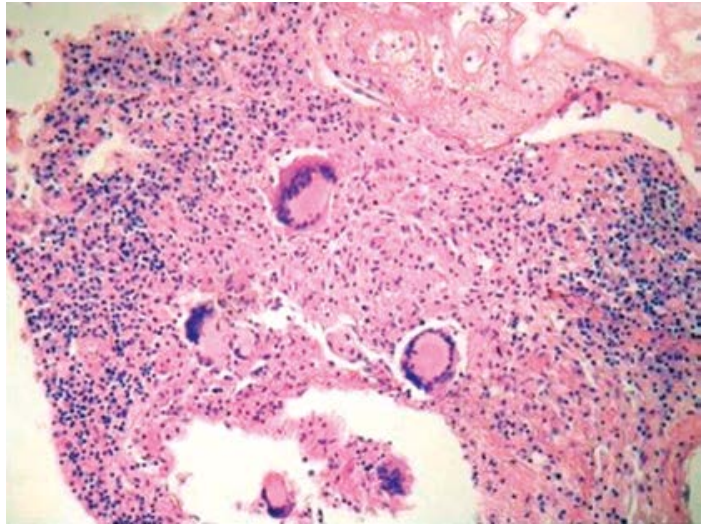

Figure 1. Histopathological picture of tubercular granulomas from the middle ear. $\mathrm{H}+\mathrm{E}$ staining

in diameter). Massive lymph nodes - about 5-6 cm in diameter - were detected in 4 cases.

Ultrasonography of the cervical lymph nodes was performed in 23 (88.5\%) cases, where presumptive diagnosis either of cyst or reactive lymphadenitis or abscess was proposed. Chest X-ray was performed in all patients and the evidence of lung tuberculosis was confirmed in 7 (27\%) cases.

In all patients the diagnosis was estimated by histopathological examination of the excised lymph node.

Fine needle biopsy was performed in 19 cases, 3 of which were negative. The remaining 16 (84\%) cases were positive, but in most of them the examining pathologist suggested excisional biopsy of the node to confirm the diagnosis.

Histopathological criteria for diagnosis of tuberculosis were granulomatous inflammation with caseous necrosis (Figure 1).

Tuberculin skin test was positive in 10 (50\%) out of 20 tests performed. Contact history with active tuberculosis was detected in 4 (15.4\%) cases.

\section{Laryngeal tuberculosis - 20 (27.4\%) cases}

The age range in this group was from 42 to 75 years. Mean age 56.5 years. There were 14 men (mean

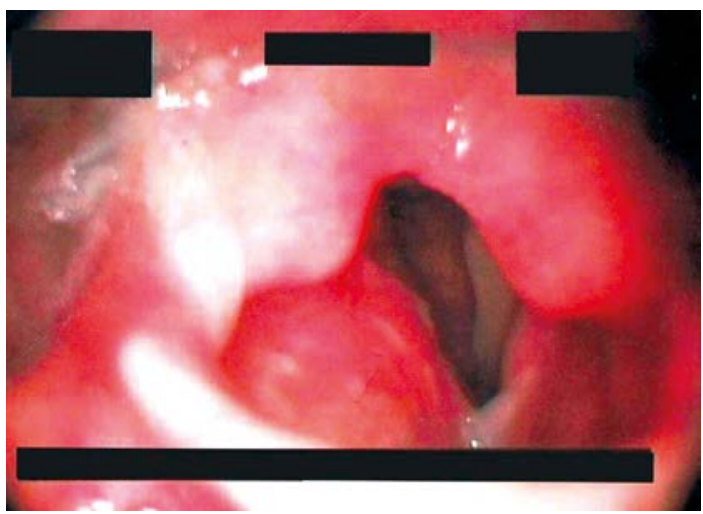

Figure 2. Appearance of laryngeal tuberculosis: thickening of the right false vocal fold and ulceration of the right arytenoid age: 57 years) and 6 women (mean age: 55.3 years). The mean time from the onset of symptoms to hospitalization was 22 weeks (range: 10-63 weeks).

The complaints at admission were as follows: hoarseness - 18 (90\%) cases; cough - 9 (47\%) cases; dysphagia - 4 (20\%) cases; dyspnea - 2 (10\%) cases; systemic symptoms (malaise, fever and weight loss) -6 (30\%) cases.

Eighty percent of patients were referred to our department with the presumptive diagnosis of laryngeal tumor. On the laryngoscopy the morphology and localization of the tumor in the larynx and the mobility of vocal folds were examined. The results are presented below:

- in $8(40 \%)$ cases we observed ulcerative tumor in the larynx, in 7 (35\%) cases papillomatous tumor, and in 5 (25\%) cases chronic hypertrophic laryngitis;

- in $12(60 \%)$ cases pathology was localized in vocal folds, in $8(40 \%)$ cases in the posterior commissure of the larynx, and in 5 (25\%) cases in vestibular folds.

- in $75 \%$ of cases there was only one localization of tumor, while in $25 \%$ there were multiple lesions in the larynx;

- $15 \%$ of patients had unilateral laryngoplegia.

In the group of laryngeal tuberculosis the diagnosis was established in all cases on histopathological examination of excised pathological tissue.

Moreover, histopathological examination revealed in 5 cases coexistence of planoepithelial carcinoma with tuberculosis. In all 5 cases total laryngectomy was performed. Chest X-ray was performed in all patients and the evidence of lung tuberculosis was confirmed in 14 (70\%) cases. Tuberculin skin test was positive in $10(66.6 \%)$ out

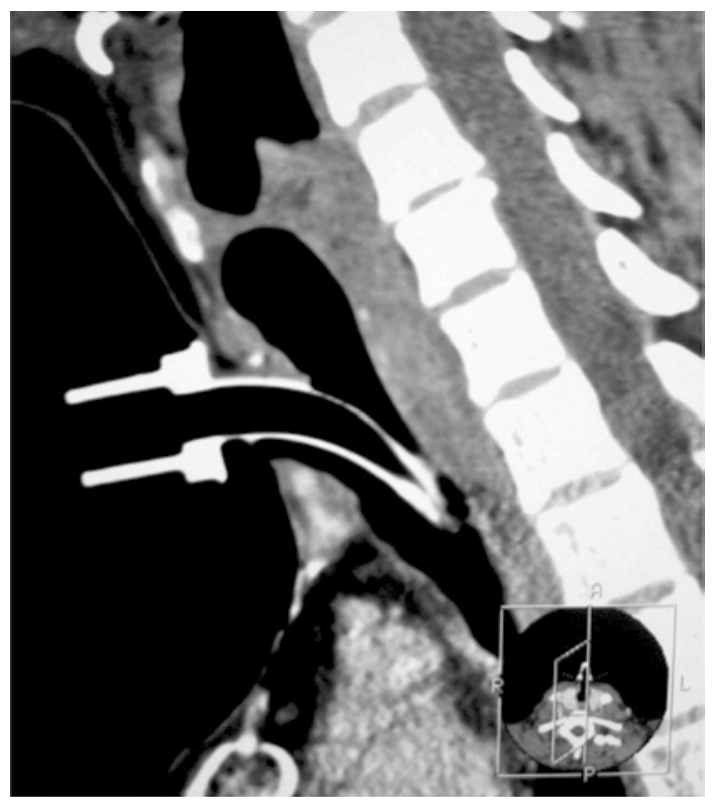

Figure 3. Laryngostenosis in patient with laryngeal tuberculosis. Tracheostomy 
of 15 tests performed. Contact history with active tuberculosis was detected in 3 (15\%) cases (Figures 2 and 3 ).

\section{Oropharyngeal tuberculosis - 10 (13.7\%) cases}

The age range in this group was from 49 to 71 years. Mean age 58.2 years. There were 7 men (mean age: 58.1 years) and 3 women (mean age: 58.3 years).

The mean time from the onset of symptoms to hospitalization was 14 weeks (range: 6-25 weeks).

The complaints at admission were as follows: ulceration in oropharynx - 4 cases; tumor in oropharynx -3 cases; treatment-resistant tonsillitis - 3 cases; dysphagia - 5 cases; sore throat 5 cases; systemic symptoms (malaise, fever and weight loss) -2 cases.

On examination the localization of pathology was established as follows: tonsils in 7 cases 4 tonsillectomies and 3 excisional biopsies were performed; soft palate in 2 cases - samples of tissue were collected; lateral surface of tongue in 1 case - sample of tissue was collected.

In all cases the diagnosis was based on histopathological examination. Furthermore, in 1 case of tonsil tuberculosis coexistence of planoepithelial carcinoma was detected and the patient was sent for both antitubercular and radiotherapy treatments.

Chest X-ray was performed in all patients and evidence of lung tuberculosis was observed in $3(30 \%)$ cases. Tuberculin skin test was positive in $3(43 \%)$ cases out of 7 tests performed. Contact history with active tuberculosis was detected in only $1(10 \%)$ case.

\section{Salivary gland tuberculosis - 9 (12.3\%) cases}

The age range in this group was from 52 to 72 years. Mean age 64.3 years. There were 3 men (mean age: 68 years) and 6 women (mean age: 62 years). The mean time from the onset of symptoms to hospitalization was 20 weeks (range: 14-36 weeks).

Within the group there were: 6 cases of parotid gland tuberculosis -4 on the left side and 2 on the right side; 3 cases of submandibular gland tuberculosis - both on the right side.

The most common complaints on admission were: swelling of the affected salivary gland region - 5 cases; tumor of the salivary gland -4 cases; pain of the salivary gland region -3 cases.

No patients suffered from systemic symptoms or facial nerve palsy.

Ultrasonography was performed in all cases. Results suggested either enlargement of the gland with a diffuse hypoechogenic area or localized hypoechogenic tumor. Ultrasonography was fol- lowed by fine needle biopsy of the pathological gland and there was a diagnosis of caseating granulomas suggestive of mycobacterial infection in 5 cases.

In 4 cases the results of fine needle biopsy were not diagnostic and these patients underwent surgical treatment. The histopathological examination of excised tissue revealed in all cases tuberculosis of the salivary tissue. Moreover, in two cases there were also described changes characteristic for tuberculosis in deep intraparenchymal nodes of the parotid gland.

Chest X-ray was performed in all patients and there was no evidence of lung tuberculosis. Tuberculin skin test was positive in 2 (30\%) cases out of 6 tests performed. Contact history with active tuberculosis was detected in 1 (11\%) case.

\section{Tuberculosis of nose and paranasal sinuses - $3(4.1 \%)$ cases}

The age range in this group was from 58 to 68 years. Mean age 61.7 years. There were 2 men (mean age: 63.5 years) and 1 woman (mean age: 58 years). The mean time from the onset of symptoms to hospitalization was 14 weeks (range: 8-18 weeks).

All 3 patients were referred to our department with the suspicion of sinonasal tumor for further diagnosis and treatment.

The complaints on admission were as follows: nasal obstruction on one side -3 cases; purulent or bloody discharge from one nostril - 2 cases; headache -2 cases; pain localized in paranasal sinuses - 1 case; exophthalmos - 1 case; systemic symptoms -1 case.

On laryngological examination in all patients there were pathological pale granulation tissues in the nasal cavity. Sinus X-ray was performed in all patients, but none of them was specific.

In 1 case Caldwell-Luc operation was performed and in the other 2 cases some granulation tissue from the nasal cavity was collected. Histopathological examination revealed tuberculosis in all cases.

One patient had changes remarkable for tuberculosis in chest X-ray. Tuberculin skin test was positive in 1 (50\%) patient out of 2 tested. There was no history of contact with active tuberculosis.

\section{Tuberculous otitis media $-3(4.1 \%)$ cases}

The age range in this group was from 52 to 71 years. Mean age 61.3 years. There were 2 men (mean age: 61.5 years) and 1 woman (mean age: 61 years). The mean time from the onset of symptoms to hospitalization was 16 weeks (range: 12-19 weeks).

The most common complaints on admission were: otorrhea -3 cases; hearing loss -3 cases; otalgia - 1 case; tinnitus - 1 case; facial nerve palsy -1 case. 


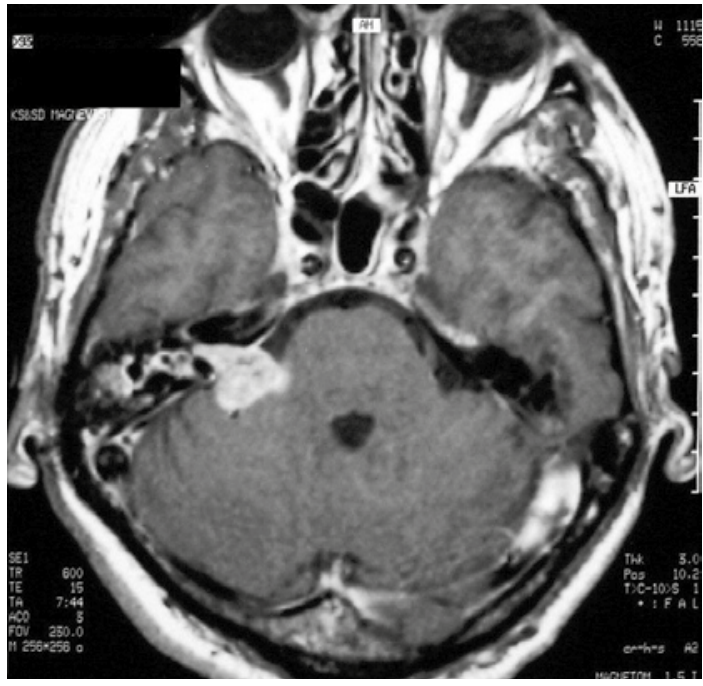

Figure 4. Computed tomography of temporal bone: pathological tissue in right middle ear, destruction of temporal bone, penetration to right posterior cranial fossa

In all 3 cases patients were directed to our department because of otorrhea resistant to standard treatment.

In otoscopic examination we found: subtotal perforation of the tympanic membrane with otorrhea -1 case; granulation tissue in the external auditory tube with otorrhea - 1 case; subtotal perforation with intratympanic epidermis -1 case.

We performed computed tomography of the temporal bone in all patients and it showed occupation of soft tissues in the middle ear and mastoiditis. Bone destruction and progression of the pathology to the apex of the temporal bone was detected in only 1 case (Figure 4).

In all 3 cases surgical treatment - tympanoplasty - was performed. The histopathological examination of granulation revealed tuberculous otitis media in all cases.

Chest X-ray of one of the patients showed some fibrosis in the apex of both lungs that might have suggested tuberculosis in the past. Tuberculin skin test was positive in 2 (66.6\%) out of 3 tests performed. There was no history of contact with active tuberculosis.

As soon as the diagnosis was established, each patient was referred for comprehensive diagnosis and treatment to one of the two specialist centers of tuberculosis treatment in Warsaw.

\section{Skin tuberculosis - 2 (2.7\%) cases}

In both cases the patients were females. The first patient, a 56-year-old woman, presented with localized swelling of tissues on the left side of the neck of 3 days duration. Skin over the swelling was reddish. Ultrasonography showed localized inflammation of soft tissue of the neck without fluid collection. Antibiotics were administered. During the next 3 days progression of inflammation of the neck tissue was observed, despite the good general condition of the patient. On the fourth day of hospitalization incision and drainage was performed. There was no discharge. In the next few days there developed a fistula with granulation in the place of incision. The histopathological examination of granulation revealed tuberculosis.

The second patient, a 78-year-old woman, was referred to our department with ulceration on the left cheek of $2 \mathrm{~cm}$ diameter and a history of 2 months, which was refractory to standard treatment with antibiotics and antifungal drugs.

On examination the ulceration was well localized, rather shallow with some granulation tissue. The rest of the laryngological examination was normal for the patient's age. Samples of tissue were collected for histopathology, which showed features of tuberculosis.

Both of the patients were directed for comprehensive diagnosis and treatment to one of the two specialist centers of tuberculosis treatment in Warsaw.

\section{Discussion}

Tuberculosis rarely occurs in otolaryngological practice, but unrecognized and without treatment is potentially lethal and remains an epidemiological threat.

In our material we found on average three cases per year of tuberculosis in the head and neck region. In the literature most of the articles concerning head and neck tuberculosis are case reports. Nalini et al. from India presented very extensive material of 117 cases from a 4-year period [3]. Authors from the United Kingdom described 23 cases of head and neck tuberculosis also from a 4-year period, but they noted that all patients were immigrants (Asian, African, Afro-Caribbean and Caucasian) [4].

For the whole group the male to female ratio was $1.43: 1$ and this confirms the observation of other researchers that tuberculosis is more common in males [5]. When we analyzed the male to female ratio in each group we found that it was similar for the group with cervical lymph node tuberculosis $(1.36: 1)$. Moreover, this ratio was remarkably higher for the group of laryngeal and oropharyngeal tuberculosis $(2.3: 1)$. In our opinion this is due to additional risk factors such as smoking and alcohol abuse, both of which in our country are more prevalent in men.

For all of the patients, the mean time that had elapsed between the onset of symptoms and hospitalization was 15 weeks and it was the longest within the group of laryngeal tuberculosis - 22 
weeks. The same long period of observation is described by other researchers [4].

Positive chest $\mathrm{X}$-ray in patients with head and neck tuberculosis varies in different studies from $14 \%$ [6] to $54 \%$ [3] and 55\% [4]. In our material pulmonary tuberculosis was identified in $35.6 \%$ of cases. The highest incidence of lung tuberculosis is observed in patients with laryngeal tuberculosis: $80-92.3 \%$ [7, 8], and in our study $70 \%$. This indicates not only hematogenous but also direct spread of bacilli from the lungs.

The radiological view of pulmonary disease included apical fibrosis, apical cavities, fibrocalcified lesions, fibrosis of the lobe, shadows and pleural thickening.

Only $12 \%$ of patients gave us a history of contact with active tuberculosis. Our study confirmed the information of low incidence ( $20 \%$ cases) of systemic symptoms in head and neck tuberculosis. However, Penfold et al. observed one of the systemic symptoms in $52 \%$ of patients [4].

Tuberculin skin test was positive only in $53 \%$ of cases, which proves poor diagnostic sensitivity of this method.

None of our patients had suffered from immunosuppressive disease or had undergone immunosuppressive treatment. The majority of the patients $(68 \%)$ were smokers, but few of them confessed to alcohol abuse. Unfortunately, the data concerning risk factors were not collected conscientiously enough and cannot be analyzed.

In Poland, testing for HIV is not routinely done in suspicion of tuberculosis. Moreover, the patient must agree to be tested for HIV. None of our patients had such testing so we are unable to determine the status of incidence of HIV in head and neck tuberculosis.

The commonest presentation of head and neck tuberculosis is cervical lymph node tuberculosis [3, 4]. Most of the cases present as a non-tender, small to moderate size, firm mass on the neck in an otherwise asymptomatic patient. In our study, we found that the majority of these patients had unilateral disease (92\%) with a single group of lymph nodes involved. We observed that in $57.7 \%$ tuberculosis was localized in deep jugular chain nodes and in $42.3 \%$ in submandibular nodes. We did not find any case of posterior triangle cervical lymph node tuberculosis, which in other reports is also a common localization of infection $[3,6]$.

Eighty-one percent of patients had normal $X$-chest rays, which confirms the results of other studies $[5,9,10]$ that most patients with tuberculosis of the lymph nodes do not have active pulmonary disease.

Ultrasonography did not prove helpful in establishing the diagnosis. Fine needle biopsy was positive in $84 \%$, but in most of the results it suggested confirmation in excisional biopsy.
For this reason we agree with other authors, who recommend excisional biopsy as the most reliable method to not delay the final diagnosis, which in this region can be very difficult, because of many other conditions that need to be differentiated [4].

The second most common localization of head and neck tuberculosis is the larynx [4]. Reports estimate that it accounts for nearly $1 \%$ of all cases of tuberculosis [11]. Symptoms and laryngoscopic appearance are not specific. Clinical manifestation can mimic many other pathological states in the larynx, especially neoplasm.

In our study, hoarseness was found to be the most common symptom ( $90 \%$ of patients), as indicated in other reports [3, 7, 8, 12-14]. The majority of our cases were single lesions (75\%), localized in true vocal folds (60\%), which in recent studies has been found to be a typical presentation of laryngeal tuberculosis $[3,7,8,13,14]$. The prevalence of involvement of the posterior larynx, so commonly reported in the past, is presently not observed.

From our observations the coexistence of carcinoma with laryngeal tuberculosis is quite remarkable (25\%). For this reason, there is always a necessity of histopathological examination of laryngeal tissue.

Oropharyngeal tuberculosis is the third localization of head and neck tuberculosis with tonsils as the most commonly affected site. Clinical manifestation, in the majority of cases, mimics malignant pathology and typically presents as a chronic, non-healing ulceration or small tumor, painful or painless [3, 15-17].

From our experience, excisional biopsy followed by histopathological examination is recommended to avoid misdiagnosis of coexisting cancer and to implement proper and immediate treatment. Salivary gland tuberculosis and tuberculosis of the paranasal sinuses usually present with symptoms that suggest neoplastic pathology [18].

Otherwise, aural tuberculosis mimics chronic otitis media, typically occurring with persistent otorrhea refractory to standard treatment, hearing loss and subtotal or total perforation of tympanic membrane with granulation tissue in the cavity and nonspecific appearance in CT scans.

Multiple perforations of the tympanic membrane as described in former reports have not been encountered in recent research [19, 20]. Even facial nerve palsy, highly suggestive of tuberculous otitis media, is currently rarely reported.

We agree that histopathological examination of infectious granulation should be performed in every case of chronic otitis media [3, 21].

From our study, we found that tuberculosis of the head and neck region is not at all casuistic pathology and no longer presents with formerly described specific symptoms. As clinicians we 
should always take it into consideration in differential diagnosis and remember that the most important diagnostic tools remain clinical evaluation and biopsy followed by histopathological examination.

In conclusion, tuberculosis still remains a problem and must be taken into consideration in the diagnostic process. The commonest presentation of head and neck tuberculosis was found to be cervical lymph node tuberculosis. The coincidence of tuberculosis and cancer is remarkable in the head and neck region. Larynx tuberculosis no longer presents with the formerly described specific clinical manifestation. Although salivary glands, paranasal sinuses and ear are rare localizations of tuberculosis, we should consider this pathology in differential diagnosis.

\section{References}

1. Global Tuberculosis Control. Surveillance, Planning, Financing. WHO Report 2008.

2. Tuberculosis still threat. http://www.katowice.uw.gov.pl/ wdznnsoz/Opracowania_SCZP_2008/printpage.html

3. Nalini B, Subramaniam V. Tuberculosis in ear, nose and throat practice: its presentation and diagnosis. Am J Otolaryngol 2006; 27: 39-45.

4. Penfold CN, Revington PJ. A review of 23 patients with tuberculosis of head and neck. Br J Oral and Maxillof Surg 1996; 34: 508-10.

5. Dietel M, Bendago M, Kraijden S. Modern management of cervical scrofula. Head Neck 1989; 11: 60-6.

6. Dharma KB, Rekesh P, Bimal KS, Ram CMA. Distribution of lymph nodes in cases of tuberculous cervical lymphadenitis. Acta Otolaryngol 2004; 124: 1095-8.

7. Chen-Chi W, Chang-Chun L, Ching-Ping W, Shih-An L, Rong-San J. Laryngeal tuberculosis: a review of 26 cases. Otolaryngol Head Neck Surg 2007; 137: 582-8.

8. Levenson MJ, Ingerman M, Grimes C, Robbett WF. Laryngeal tuberculosis:Review of twenty cases. Larygoscope 1984; 94: 1094-7.

9. Olu Ibekwe A, Al Shareef Z, Al Kindy S. Diagnostic problems of tuberculous cervical adenitis (scrofula). Am J Otolaryngol 1997; 18: 202-5.

10. Reed DL, Bergeron RT. Cervical tuberculosis adenitis: CT manifestations. Radiology 1985; 154: 701-804.

11. Rizzo PB, Da Mosto MC, Clari M, Scotton PG, Vaglia A, Marchiori C. Laryngeal tuberculosis: an often forgotten diagnosis. Int J Infect Dis 2003; 7: 129-31

12. Kandiloros DC, Nikolopoulos TP, Ferekidis EA. Laryngeal tuberculosis at the end of 20th century. J Laryngol Otol 1997; 11: 619-21.

13. Bailey CM, Windle-Taylor PC. Tuberculous laryngitis: a series of 37 patients. Laryngoscope 1981; 91: 93-100.

14. Kulkarnni NS, Gopal GS, Ghaisas SG, Gupte NA, Phil M. Epidemiological considerations and clinical features of ENT tuberculosis. J Laryngol Otol 2001; 115: 555-8.

15. Miziara ID. Tuberculosis affecting the oral cavity in Brazilian HIV-infected patients. Oral Surg Oral Med Oral Pathol Oral Radiol Endod 2005; 100: 179-82.

16. Sareen D, Sethi A, Agarwal AK. Primary tuberculosis of the tongue: a rare nodular presentation. Br Dent J 2006; 200: $321-2$
17. Michalak A, Wojtas G, Kidawa I, Tylżanowska-Nitek K. Tuberculosis of tongue in patient with disseminated pulmonary tuberculosis. Pneumonol Alergol Pol 2004; 72: 28-31.

18. Sethi A, Sareen D, Sabherwal A, Malhotra V. Primary parotid tuberculosis: varied clinical presentation. Oral Dis 2006; 12: 213-5.

19. Meher R, Singh I, Yadav SPS, Gathwala G. Tubercular otitis media in children. Otolaryngol Head Neck Surg 2006; 135: 650-2.

20. Vaamonde P, Castoro C, Garcia-Soto N, Labella T. Tuberculous otitis media: a significant diagnostics challenge. Otolaryngol Head Neck Surg 2004; 130: 759-66.

21. Bruzgielewicz A, Wysocki J, Osuch-Wójcikiewicz E. Head and neck tuberculosis - still up-to-date problem. Otolaryng Pol 1995; 48: 566-73. 